\title{
Staphylococcus aureus intra-mammary infection affects the expression pattern of IL-R8 in goat
}

\author{
Filipe J. ${ }^{\mathrm{a}}$, Bronzo V. ${ }^{\mathrm{a}}$, Curone G. ${ }^{\mathrm{a}}$, Castiglioni B. ${ }^{\mathrm{b}}$, Vigo D. ${ }^{\mathrm{a}}$, Smith B. ${ }^{\mathrm{c}}$, Herrera V. ${ }^{\mathrm{a}}$, Roccabianca P. ${ }^{\mathrm{a}}$, \\ Moroni P. ${ }^{\mathrm{a}, \mathrm{d}}$, Riva F. ${ }^{\mathrm{a}}$ * \\ ${ }^{a}$ Università degli Studi di Milano, Dipartimento di Medicina Veterinaria, via Celoria 10, 20133, Milan, Italy \\ b Istituto di Biologia e Biotecnologia Agraria, Consiglio Nazionale delle Ricerche, 26900, Lodi, Italy \\ ${ }^{\mathrm{c}}$ University of California, Davis, Wildlife Health Center, Davis, CA 95616, USA \\ d Cornell University, Animal Health Diagnostic Center, Quality Milk Production Services, Ithaca, NY 14853, USA
}

\section{A R T I C L E IN F O}

\section{Keywords:}

IL-1R8/TIR8/SIGIRR

Staphylococcus aureus

Intra-mammary infection

Goat

Innate immunity

\begin{abstract}
A B S T R A C T
IL-1R8 is a member of Interleukin-1 receptor family acting as a negative regulator of inflammation reliant on ILRs and TLRs activation. IL-1R8 role has never been evaluated in acute bacterial mastitis. We first investigated IL-1R8 sequence conservation among different species and its pattern of expression in a wide panel of organs from healthy goats. Then, modulation of IL-1R8 during natural and experimental mammary infection was evaluated and compared in blood, milk and mammary tissues from healthy and Staphylococcus aureus infected goats. IL-1R8 has a highly conserved sequence among vertebrates. Goat IL-1R8 was ubiquitously expressed in epithelial and lymphoid tissues with highest levels in pancreas. IL-1R8 was down-regulated in epithelial mammary cells following $S$. aureus infection. Interestingly it was up-regulated in leukocytes infiltrating the infected mammary tissues suggesting that it could represent a target of $S$. aureus immune evasion.
\end{abstract}

\section{Introduction}

In small ruminant dairies mastitis represents one of the most relevant health issues causing the reduction of milk production, high veterinary care costs with increased use of antibiotics and animal culling that are responsible for severe economic losses [1]. Mastitis is mainly caused by contagious and/or environmental microorganisms leading to overt clinical or sub-clinical episodes. In small ruminants the most frequently isolated pathogen in clinical cases is Staphylococcus aureus [2]. Once S. aureus invades the mammary gland, it is sensed by epithelial cells through their Pattern Recognition Receptors (PRRs). These receptors bind to the Pathogen Associated Molecular Patterns (PAMPs) triggering a signaling cascade leading to the establishment of an inflammatory response accompanied by leukocyte recruitment that is the basis for pathogen clearance [3-5].
The most important PRRs are Toll Like Receptors (TLRs) that are widely expressed by several cell types and tissues. TLRs belong to a superfamily that includes also the Interleukin-1 like receptors (ILRs). The members of the superfamily are characterized by the presence of the conserved cytoplasmic TIR domain involved in the signal transduction [6]. Upon ligand binding, the ILRs and TLRs dimerize through their TIR domain and recruit the TIR domain of a cytoplasmic adapter molecule, such as MyD88, forming a receptor complex. The signaling cascade involves also other molecules such as IRAK, TRAF6 and leads to downstream activation of NF-kB or other transcription factors such as AP-1, JNK, and IRF $[7,8]$. The activation of different transcription factors drives the expression of pro-inflammatory cytokines and chemokines and the expression cell survival and proliferation genes [9]. The activation of immune and inflammatory responses by TLR and ILR engagement in, such as in sepsis and chronic inflammation, is potentially detrimental for the organism [10]. In order to avoid severe tis-

Abbreviations: IL-1R8, Interleukin-1 receptor 8; MEC, Mammary epithelial cells; PMNs, Polymorphonuclear leukocytes; TLRs, Toll like receptors; SCC, Somatic cell count; PRMs, Pat-

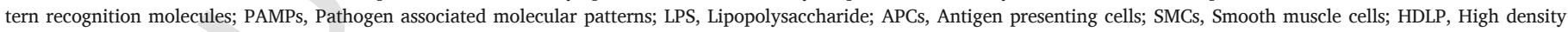
lipoprotein; MFG, Milk fat globules.

* Corresponding author.

Email addresses: joel.soares@unimi.it (J. Filipe ); valerio.bronzo@unimi.it (V. Bronzo ); giulio.curone@unimi.it (G. Curone ); casti@ibba.cnr.it (B. Castiglioni ); daniele.vigo@unimi.

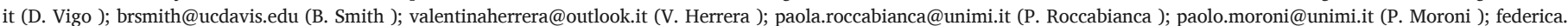
riva@unimi.it (F. Riva) 
sue damages caused by an exaggerated inflammatory response, the activity of ILRs and TLRs is tightly regulated at different levels including receptor antagonists (IL-1RA), decoy receptors (IL-1R2), soluble receptors (sST2), decoy signaling molecules (MyD88s) and miRNAs (miR-155) that regulate translation of the mRNAs [10]. IL-1R8, also known as TIR8 or SIGIRR, is a decoy receptor with a negative regulatory activity on TLRs and ILRs. It is a fringe member of the ILR family that presents three main differences compared to the other family members: a single extracellular Ig domain (instead of three), an unusual 95 residue long tail, and a TIR domain lacking two conserved residues [11]. Its nucleotide sequence and pattern of expression are conserved among vertebrates [12]. In mouse and human IL-1R8 is expressed in epithelial tissues (e.g. kidney, gastrointestinal tract, liver, lung), in lymphoid organs and in leukocytes (monocytes, lymphocytes, DCs, NK cells) $[13,14]$. The mechanisms of the regulation of IL-1R8 expression are still unclear, but down modulation has been described in several inflammatory conditions [15-22]. Stimuli inducing IL-1R8 expression are still unknown.

IL-1R8 has been demonstrated to unroll an inhibitory activity on IL-1R1, IL-1R5 (IL-18R), IL-1R4 (T1/ST2), TLR1/2, TLR3, TLR4, TLR7, and TLR9 [23-28]. The mechanism of inhibition exerted by IL-1R8 involves both its extracellular and intracellular domains. Indeed, IL-1R8 is recruited at the receptor complex site of TLR4 and TLR7 and interferes with the dimerization of MyD88. Specifically, IL-1R8 binds the TIR domain of TLR4 and TLR7 through a BB-loop in its own TIR domain that disturbs the MyD88 pocket and reduces the activation of the downstream signaling molecules [29]. The extracellular domain of IL-1R8 was demonstrated to prevent the dimerization of IL-1R1, IL-1R3, and to block the activation of IL-1R4 [23,24]. In conclusion, IL-1R8 can inhibit the activation of different transcription factors induced by TLRs and ILRs, such as NF-kB, JNK and mTOR [30,31].

Given the anti-inflammatory role of IL-1R8, its modulation in mammary gland could represent a novel strategy of immunotherapy for mastitis prevention and treatment, reducing tissue damage and the use of antibiotics.

In this work, the pattern of IL-1R8 expression was evaluated in caprine healthy organs and tissues and its modulation during $S$. aureus intra-mammary infection was investigated.

\section{Material and methods}

\subsection{Animals and samples}

In this study three groups of samples were used: a) organ and tissue samples from two healthy goats, b) archived mammary tissues from six goats experimentally infected with $S$. aureus from a previous study by Cremonesi et al. 2012 [32], c) milk and blood samples from ten lactating goats. The study complied with the recent Italian animal experimentation (D. Lgs. 26/2014) and ethics (Italian Health Ministry authorization n. 628/2016-PR) laws.

Samples from a wide panel of tissues and organs (a) were taken from two healthy goats at the slaughterhouse. A $0.5 \mathrm{~cm}^{3}$ tissue sample was taken from each organ following standard procedures at slaughterhouse and immediately immersed in $3 \mathrm{ml}$ of RNAlater (Life Technologies, Carlsbad, CA, USA), and stored at $-20^{\circ} \mathrm{C}$ until use.

Archival mammary tissue samples from six goats, experimentally infected with $S$. aureus in a previous study [32], were collected and analyzed $30 \mathrm{~h}$ post infection (b).

Finally, $15 \mathrm{ml}$ of composite milk and $20 \mathrm{ml}$ of blood were collected from nine goats ( 6 healthy goats and 4 goats with clinical $S$. aureus mastitis), located at Roccaforte Ligure (Alessandria, Italy). Before milk sampling, teat ends were carefully cleaned. First streams of foremilk were discharged, and then approximately $15 \mathrm{ml}$ of milk were collected aseptically into sterile vials. Milk was transported at $4{ }^{\circ} \mathrm{C}$ to the labora- tory and immediately processed. $S$. aureus infection was diagnosed following NMC procedure [33]. Peripheral blood was collected from the jugular vein in vacutainer tubes containing EDTA (BD, Franklin Lakes, New Jersey, USA), and transported to the laboratory at room temperature to avoid neutrophil activation.

\subsection{Immunohistochemistry}

Formalin fixed, routinely processed and paraffin embedded archived mammary tissue samples [32] were cut in $3 \mu \mathrm{m}$ slices and affixed on the slide. Following immersion in xylene and descending alcohols, as previously described [34], samples were blocked with $10 \%$ heat inactivated horse serum and incubated $18 \mathrm{~h}$ at $4{ }^{\circ} \mathrm{C}$ with the anti-human SIGIRR primary polyclonal antibody (AF990, R\&D Systems, Minneapolis, MN, USA) antibody, at 1:1000 dilution in TRIS buffer. Following three washes in TRIS buffer for $10 \mathrm{~min}$ each, samples were incubated $30 \mathrm{~min}$ at room temperature with anti-goat biotinylated antibody made in horse (IgG, Dako, Glostrup, Denmark) at 1:200 dilution. Finally, slides were incubated with avidin-biotin reagent (Vectastain Elite ABC system, Vector, Burlingame, CA, USA) for $30 \mathrm{~min}$. Signal detection was made using 3-amino-9-ethylcarbazole (AEC, vector kit, Burlingame, CA, USA) as peroxidase substrate for $15 \mathrm{~min}$, followed by a Mayer's hematoxylin counter stain (Sigma-Aldrich, St. Louis, USA). After wash the slides were coverslipped and stored at room temperature for microscopical evaluation. Anti-human SIGIRR antibody is polyclonal and recognizes multiple different epitopes of the target protein that are not only conformational but also sequential (around 70-90 kD). Moreover SIGIRR, also known as IL-1R8and/or TIR8, has a highly conserved sequence (DNA and Protein) as described previously [12] and in the present manuscript. Based on these observations and on the previously published data $[35,36]$ the polyclonal anti human SIGIRR antibody is cross reactive at least with tome epitopes of the goat IL-1R8.

\subsection{Milk processing}

Milk fat globules (MFG) and milk cells were isolated as previously described [34]. Briefly, milk was centrifuged and $500 \mu \mathrm{l}$ of surface fat layer (MFG) were added to a sterile $2 \mathrm{ml}$ tube containing TRIzol (Invitrogen, Life Technologies, Carlsbad, CA, USA). The samples were kept at $-80^{\circ} \mathrm{C}$ until use.

Skimmed milk was eliminated and the cell pellet was resuspended in $1 \mathrm{ml}$ of RPMI (Invitrogen, Life Technologies, Carlsbad, CA, USA) supplemented with 10\% FCS (Invitrogen, Life Technologies, Carlsbad, CA, USA). The cells $\left(0.5-2 \times 10^{6}\right)$ were lysed in TRIzol (Invitrogen, Life Technologies, Carlsbad, CA, USA) and then stored at $-80^{\circ} \mathrm{C}$.

\subsection{Blood cells isolation}

Blood cells were isolated as previously described [34]. Briefly, blood $(1 \mathrm{ml})$ was lysed in ACK hypotonic solution $\left(0.5 \mathrm{M} \mathrm{NH} \mathrm{N}_{4} \mathrm{Cl}, 10 \mathrm{mM}\right.$ $\mathrm{KHCO}_{3}, 200 \mu \mathrm{l} 0.5 \mathrm{M}$ EDTA pH 8). After wash, the cells were resuspended RPMI (Invitrogen, Life Technologies, Carlsbad, CA, USA) supplemented with $10 \%$ FCS. $10^{6}$ cells were used for cytospin preparation, while $2-3 \times 10^{6}$ cells were lysed in TRIzol (Invitrogen, Life Technologies, Carlsbad, CA, USA) and stored at $-20^{\circ} \mathrm{C}$.

The rest of the blood samples $(19 \mathrm{ml})$ was diluted $1: 2$ in sterile PBS (Sigma-Aldrich. St. Louis, USA), and layered over a cushion of $10 \mathrm{ml}$ of Histopaque-1.077 (Sigma-Aldrich. St. Louis, USA). After centrifugation, the mononuclear cells (PBMCs) ring was collected, washed in PBS (Sigma-Aldrich, St. Louis, USA), and resuspended in RPMI (Invitrogen, Life Technologies, Carlsbad, CA, USA) supplemented with 10\% FCS. The PMNs layer was recovered and transferred into a sterile tube. Red blood cells were lysed using ACK hypotonic solution. After centrifuga- 
tion, the PMNs pellet was resuspended in RPMI (Invitrogen, Life Technologies, Carlsbad, CA, USA) supplemented with 10\% FCS. Mononuclear and PMNs (5-8 $\times 10^{6}$ cells) were lysed in TRIzol (Invitrogen, Life Technologies, Carlsbad, CA, USA) and then stored at $-20^{\circ} \mathrm{C}$. The purity of PMNs and PBMCs was assessed by differential count on cytospin samples stained with H\&E. The percentage of purity of isolated PMNs resulted 91\%, whereas the one of PBMCs was $76 \%$.

\subsection{RNA extraction (from tissues and from blood and milk cells)}

Total RNA was extracted from all the samples lysed in TRIzol (mammary tissues, MFG, milk and blood cells; mononuclear and PMN cells) according to the manufacturer's protocol (Sigma-Aldrich, St. Louis, MO, USA). Finally, the RNA concentration and quality were determined using a spectrophotometer (BioPhotometer, Eppendorf, Hamburg, Germany) at $260 / 280 \mathrm{~nm}$ wavelength. The samples were stored at $-80^{\circ} \mathrm{C}$.

\subsection{Mammary tissue RNA extraction and array hybridization}

Total RNA was extracted from tissue samples as previously described [37]. RNA quality and quantity were assessed by the Bioanalyser 2100 (Agilent, Santa Clara, CA, USA). RNA samples were hybridized on a custom array (Combimatrix CustomArray $90 \mathrm{~K}$, Seattle, WA, USA) following the protocols of the manufacturer. The hybridized arrays were scanned with a GenePix 4000B microarray scanner (Axon, Toronto, Canada) and the images were exported to the Microarray Imager Software (CombiMatrix Corp., Mukilteo, WA, USA). Data were extracted and loaded into the $\mathrm{R}$ software using the Limma analysis package from Bioconductor. Systemic identification and grouping of differentially expressed genes into biological networks were performed using the software packages Ingenuity Pathway Analysis (Qiagen, Hilden, Germany).

\subsection{Reverse-transcription and real time PCR}

Total RNA $(1 \mu \mathrm{g})$ from each sample was reverse-transcribed using the High Capacity cDNA Reverse Transcription Kit (Applied Biosystems, Foster City, CA, USA), according to the manufacturer's instructions. The cDNA obtained from each sample was used as a template for Real Time PCR in an optimized $25 \mu 1$ reaction volume in MicroAmp optical 96-well plates, as previously described [37]. Goat IL-1R8 primers were designed with Primer Express software (Applied Biosystems, Foster City, CA, USA) based on the goat sequence variant 1 (GI:1062947811), but they also recognize the variant 2 and 3 (GI:1062947813 and GI:1062947815 respectively). In order to analyse the IL-1R8 gene expression we used three reference genes: glyceraldehyde-3-phosphate dehydrogenase (GAPDH), ubiquitously expressed transcript (UXT) [38] and glucose-6-phosphate dehydrogenase (G6PD) [39]. The primers for goat IL-1R8 target gene and for bovine GAPDH, bovine UXT and goat G6PD housekeeping genes [36] were purchased from ThermoFisher Scientific (Carlsbad, CA, USA). Bovine primers were used because they also specifically recognize goat sequences of GAPDH (GI:1062975189) and UXT (GI:926728962). Their sequences are listed in Table 1.

A duplicate no-template control (NTC) was also included in each plate. Real Time quantitative PCR was carried out in the 7000 Sequence Detection System (Applied Biosystems, Foster City, CA, USA) at the following thermal cycle conditions, $10 \mathrm{~min}$ at $95^{\circ} \mathrm{C}$ followed by 40 cycles of $15 \mathrm{~s}$ at $95^{\circ} \mathrm{C}$ and $1 \mathrm{~min}$ at $60^{\circ} \mathrm{C}$. Quantitation was determined after the application of an algorithm to the data analyzed by the software of the 7000 Detection System (Applied Biosystems, Foster City, CA, USA).
Table 1

Primer list.

\begin{tabular}{ll}
\hline Primer & Sequence (5'-3') \\
\hline $\begin{array}{l}\text { goat IL-1R8 for } \\
\text { goat IL-1R8 rev }\end{array}$ & GGAGGTGGAGATGAACGACG \\
goat G6PD for & GCTGCGGCTTCAGGATGA \\
goat G6PD rev & TGACCTATGGCAACCGATACAA \\
bovine GAPDH for & CCGCAAAAGACATCCAGGAT \\
bovine GAPDH rev & GGCGTGAACCACGAGAAGTATAA \\
bovine UXT for & CCCTCCACGATGCCAAAGT \\
bovine UXT rev & TGTGGCCCTTGGATATGGTT \\
\hline
\end{tabular}

The expression of IL-1R8 gene was normalized using the calculated GAPDH, UXT and G6PD average cDNA expression (mean) of the same sample and run. Moreover, efficiency of the PCR assay was shown to be $100 \%$ for the IL-1R8 (slope $=-3.3$ ), $94.4 \%$ for the GAPDH (slope $=-3.46$ ), 103\% for the UXT (slope $=-3.24$ ) and $94.6 \%$ for the G6PD (slope $=-3,45$ ) primer pairs. Qbase + software (Biogazelle, Zwijnaarde, Belgium; www.qbaseplus.com) stability analysis suggested the use of the 3 references genes. The most stable housekeeping gene resulted to be GAPDH.

\subsection{Sequence analysis}

For the analysis of the goat IL-1R8 nucleotide sequence we used free online Blast software (https://blast.ncbi.nlm.nih.gov/Blast.cgi) and for the phylogenetic tree building we used the free software on the http:// www.phylogeny.fr/simple_phylogeny.cgi website [40].

\subsection{Statistical analysis}

To compare the expression of IL-1R8 in the two experimental groups (experimentally infected and control) in the immunohistochemistry experiments we used the $t$ Student (for normally distributed data; Shapiro-Wilk test) or Mann-Whitney (for not normally distributed data; Shapiro-Wilk test) test, considering statistically significant value of $\mathrm{p}<0.05$.

To evaluate the significance of the IL-1R8 RNA messenger expression decrease in the infected mammary tissues compared to those six healthy samples, we used the Mann-Whitney test, considering statistically significant value of $\mathrm{p}<0.05$.

To compare the expression of IL-1R8 in the two experimental groups (naturally infected and healthy) of blood and milk cells and MFG, we used the $t$ Student test with Welch correction, considering statistically significant value of $\mathrm{p}<0.05$.

For the biological function and network analysis, the p-value significance calculated by right-tailed Fisher's exact test was used.

\section{Results}

\subsection{IL-1R8 nucleotide sequence is highly conserved among the species}

IL-1R8 nucleotide sequence is conserved among domestic animal species [12]. Here we further investigated the homology of IL-1R8 nucleotide sequence among a wider range of animals.

In the databases we found 3 predicted sequences of goat IL-1R8 messenger: variant 1 (GI: 1062947811), variant 2 (GI: 1062947813), and variant 3 (GI: 1062947815). These records are derived from a genomic sequence (NC_030836.1) annotated using gene prediction method, Gnomon supported by mRNA and EST evidence.

Goat IL-1R8 variant 1 sequence is homologous to several sequences of the NCBI database belonging to different species (some of them being predicted from genomic sequences), showing a very high homology 
with IL-1R8 sequence of sheep (99\%; GI:965908719), cow (96\%; GI:982972360), water buffalo (96\%; GI:594045006) and deer (95\%; GI:1187629608). A lower homology was found with sperm whale (93\%; GI:593759914), pig (91\%; GI:937575883), killer whale (90\%; GI:821405233), camel (88\%; GI:744624704), monk seal (88\%; GI:1212194754), leopard (86\%; GI:1111234795), cat (85\%; GI:1304934644), human (85\%; GI:205277450), macaque (85\%; GI:966983090), horse (84\%; GI:1333567909), mouse (79\%; GI:142374281), chicken (69\%; GI:313747564), zebrafish (68\%; GI:148356269) and xenopus (65\%; GI:1062884104) sequences.

The alignment between the unique genomic sequence (GI:1060061386) for goat IL-1R8 and the predicted mRNA variant 1 sequence, revealed that the gene of this molecule is located on goat chromosome 29 and includes ten exons as described in human [41]. Goat variant 1 and 2 are very similar in length (1791 bp vs 1778 bp, respectively) and nucleotide sequence ( $97 \%$ homology). The difference is that exons 6 of variant 2 is shorter compared to the one of variant 1 . Variant 1 and 3 are 100\% homologous from exon 2 to exon 10, but they present a completely different exon 1 .

The phylogenetic study confirmed that IL-1R8 is a conserved molecule, present from the amphibians to mammals. It is interesting to note that IL-1R8 of ruminants (cattle, goat, sheep, deer, buffalo and yak), pigs, camelids and cetaceans (dolphin and orca) has a strong homology (Fig. 1). Goat IL-1R8 sequence shows a lower homology with felines (leopard and cat), horse, bats, pinnipeds (seal and walrus), and primates (humans, orangutan, and chimpanzees), but seems quite distant from mouse (Fig. 1). As expected the sequences of chicken and xenopus

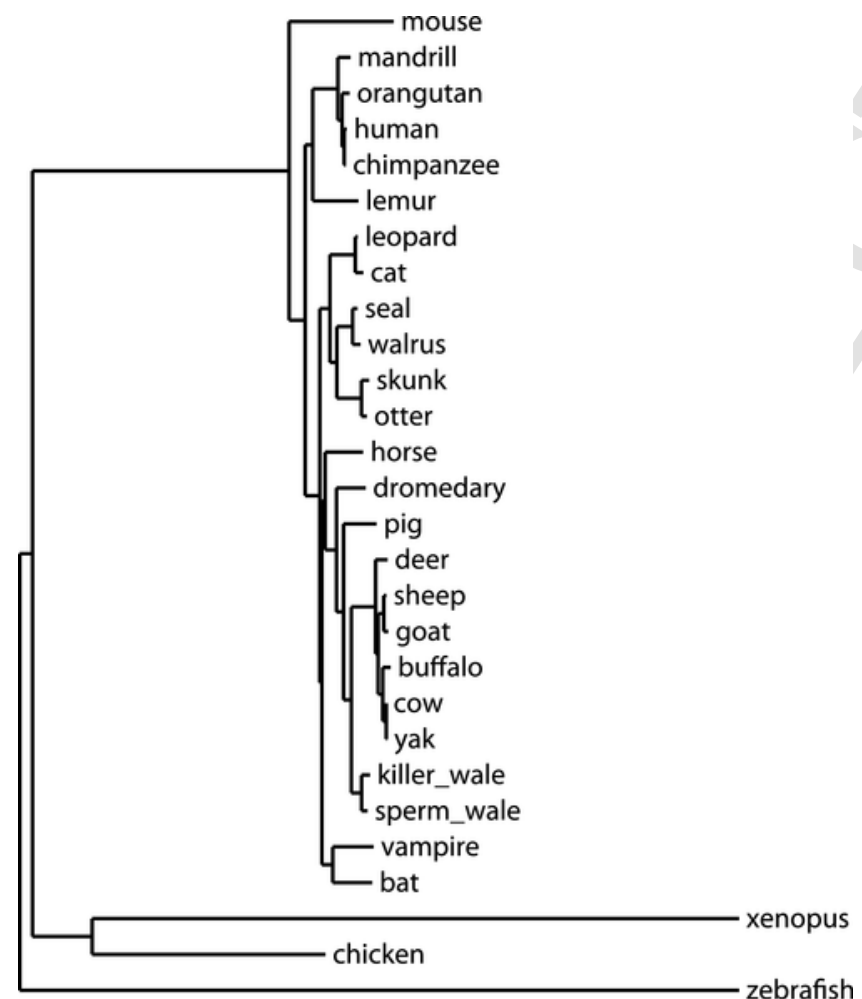

0.6

Fig. 1. Phylogenetic tree of IL-1R8 mRNA.Phylogenetic tree obtained after IL-1R8 mRNA sequences alignment of several species, using the free software on the http: //www.phylogeny.fr/simple_phylogeny.cgi web site. This cladrogram represents a Neighbour-joining tree without distances corrections (Dereeper A., Guignon V., Blanc G., Audic S., Buffet S., Chevenet F., Dufayard J.F., Guindon S., Lefort V., Lescot M., Claverie J.M., Gascuel O. Phylogeny.fr: robust phylogenetic analysis for the non-specialist. Nucleic Acids Res. 2008 Jul 1;36(Web Server issue):W465-9. Epub 2008 Apr 19). were the farthest, still having some relationship with the mammalian sequences, compared with the zebrafish one, that is the outgroup, phylogenetically earlier developed (Fig. 1).

\subsection{IL-18 is ubiquitously expressed in goat healthy organs and tissues}

Given that no data on the expression of IL-1R8 in goat were available, we first studied the expression pattern of its messenger RNA by qPCR in a large panel of organs and tissues from healthy animals.

As shown in Fig. 2 A, the mRNA expression level of IL-1R8 is very high in the liver, similarly to cattle [37]. Lower levels of expression were evident in the pancreas, kidney, lymph node, salivary gland, thymus, spleen, jejunum, adrenal gland, gastro intestinal tract (abomasum small intestine, colon), trachea, mammary gland, caecum, lung. The expression of IL-1R8 lowers in the following organs: omasum, skin, rectum, heart, bladder and rumen. Very low levels of expression were found in aorta, testis, bone marrow, CNS and esophagus.

Blood cells of healthy animals express discrete levels of IL-1R8 messenger, with a high variability among subjects (Fig. 2 B). In milk samples from healthy animals, we observed lower levels of IL-1R8 mRNA expression, in both MFG (representing epithelial mammary gland cells) and milk cells, compared to the blood cells (Fig. 2C).

\subsection{S. aureus infection results in down-regulation of $I L-1 R 8$ in the udder}

IL-1R8 plays an important role in the negative regulation of the inflammatory response dependent on TLRs and ILRs activation. Given that during clinical mastitis, major damage to the mammary tissue is ascribed to the severe inflammation, we studied the modulation of IL-1R8 in a previous experimental intra-mammary $S$. aureus infection model [32]. Six goats without any signs of mastitis were inoculated with the $S$. aureus in the left half udder and with sterile PBS, as control, in the right half udder. Thirty hours post infection (pi), goats were sacrificed and biopsies from both half udders ( 3 biopsies from each half udder: superficial, mid and deep) were taken and preserved for molecular and histological examinations [32].

IL-1R8 gene expression was analyzed by Real Time PCR in tissue samples taken at the end of the trial. In all the animals we observed a significant down-regulation of IL-1R8 in the infected udder tissue, compared to the contralateral inoculated with PBS (Fig. 3A; $p=0.041$ ).

The immunohistochemical analysis with anti-hIL-1R8 polyclonal antibody on the same tissues demonstrated that, in the control tissues, IL-1R8 is low to absent in the secretum (Fig. 3B) but is highly expressed by ductal epithelial cells: an average of $50-75 \%$ of the ductal epithelial cells resulted intensely positive (Fig. 3C and D; Supplementary Fig. 1).

In the infected tissues, vascular and acute inflammatory changes were prominent, and the expression of IL-1R8 in ductal epithelial cells was significantly down-modulated, both in terms of number of positive cells and expression intensity of the positive cells (Fig. 3C and D; Supplementary Fig. 1). The glandular epithelium didn't show any modulation in both infected and control udders, remaining slightly positive (Fig. 3C and D; Supplementary Fig. 1). Interestingly, positivity and intensity of IL-1R8 expression ( $p<0.0001$ and $\mathrm{p}<0.0001$ respectively) of leukocyte infiltrating cells were significantly increased in the infected tissues (Fig. 3C and D; Supplementary Fig. 1). The positivity of IL-1R8 expression ( $p=0.0071)$ of antigen presenting cells was significantly increased in the infected tissues (Fig. 3C). The secretum didn't show any modulation in both infected and control udders, remaining slightly positive (Fig. 3C and D). The PMNs remained negative both in the control and infected udders.

Interestingly, the Ingenuity Upstream Regulator Analysis in IPA predicts an increased activation of TLR4 pathway in the infected compared to the control udders, with the activation of the IL- $1 \beta$ and TNF 

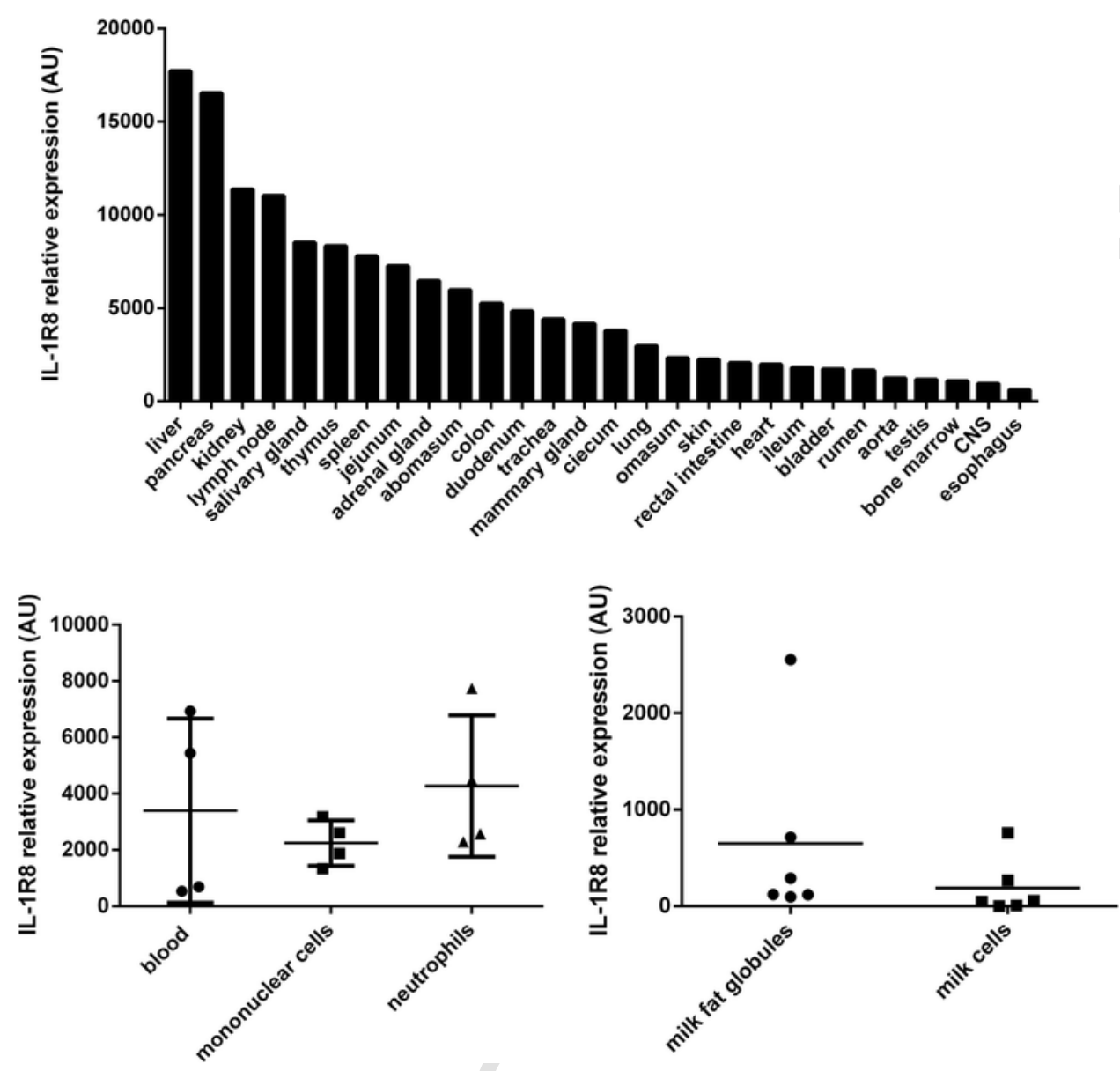

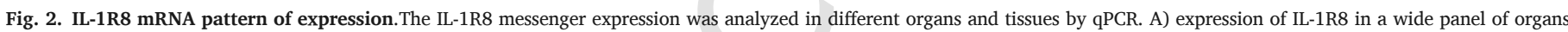

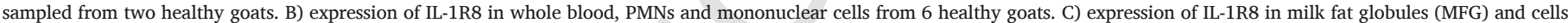

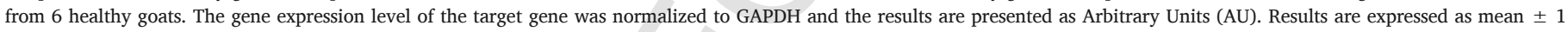
standard error in all the panels.

production. The activation of this pathway leads directly or indirectly to the increased expression of IL-18BP, ICAM1, S100A9, C3, PTX3, SOD2 and EGR1, seven of the top ten expressed genes (Fig. 4A) [34]. In order to better understand the activated pathways involved in the mammary gland response to $S$. aureus, we performed the Ingenuity Upstream Regulator Analysis in IPA in the 3 goats with the highest SCC count in milk. As shown in Fig. 4B the TLR4 pathway was confirmed to be highly activated, involving MyD88, TRAF6, NF-kB and driving to the production of IL-1 $\beta$. This predicted pathway is based on the significant statistical increased expression of several genes in the infected glands, such as: CD14 (co-receptor of TLR4), CXCL8, CCL2, CXCL2, ICAM1, NFKBIZ, NFKB1, NFKBIA, EGR1, ATF3, CSF3, IL-18BP (Fig. 4B).

\subsection{During natural S. aureus infection IL-1R8 is down-modulated in milk fat globules}

In order to study the modulation of IL-1R8 messenger in milk and blood cells during $S$. aureus infection, we collected samples from healthy and naturally infected goats. IL-1R8 was significantly down-regulated in MFG of animals affected by $S$. aureus natural infection, compared to MFG of healthy animals $(\mathrm{p}=0.0025)$. Comparison of blood and milk cells from animals positive to $S$. aureus to those from healthy animals revealed that IL-1R8 mRNA was not modulated, although a slightly trend of down-regulation seemed to be present during infection in the circulating blood PMNs and mononuclear cells, and also in milk cells (Fig. 5).

\section{Discussion}

Intra-mammary infections lead to the early activation of the innate immune response. Specifically, MEC and leukocytes can sense microbes through their PRRs and initiate an immune response to avoid infections. Activation of the inflammatory response through PRRs is fundamental to fight microorganisms because it leads to cellular responses including activation of antimicrobial killing mechanisms, production of cytokines and chemokines, maturation of antigen presenting cells, and the recruitment of the adaptive immune response. On the other hand, prolonged and uncontrolled TLRs activation could be detrimental for the tissues, resulting in an excessive inflammatory response with tissue destruction and tissue repair leading to scar and loss of function. For this reason, a complex multilayer network of mechanisms tightly regulates the activation of TLRs and ILRs at several levels by receptor antagonists (IL-1RA), decoy receptors (IL-1R2 and IL-1R8), soluble receptors (sST2 and sTLR4), decoy signaling molecules (MyD88s and IRAK-M) and miRNAs [42-44]. In the present study we focused on IL-1R8, a member of IL1R/TLR family with a negative regulatory activity on the other members of the family: TLR2, TLR4, TLR3, TLR7, TLR9, IL-1R1, IL-18R [41]. Given that no data are available on goat IL-1R8, we first searched for IL-1R8 transcript sequences in the data bases. We found 3 predicted sequences, based on genomic sequences. As in human the three sequences could represent 3 different alternatively spliced variants. Indeed there is evidence of the existence of different isoforms of 
A

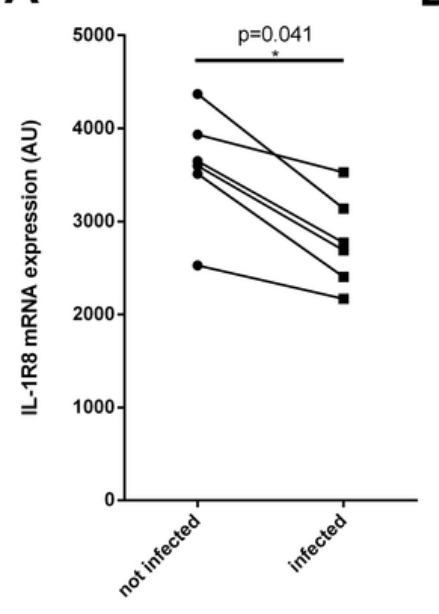

B

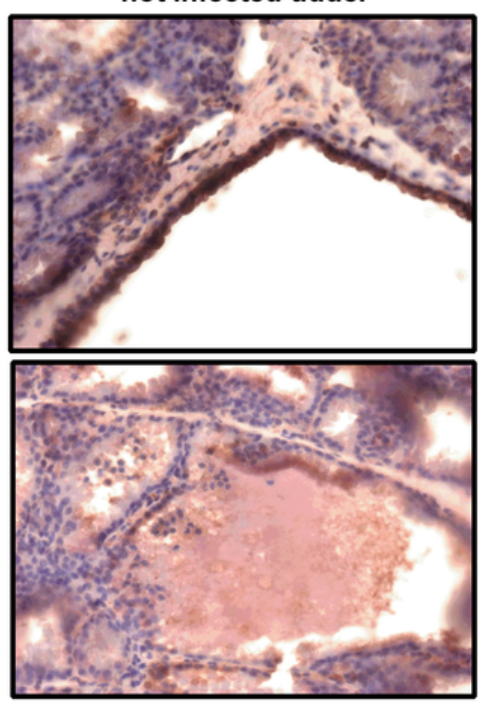

infected udder

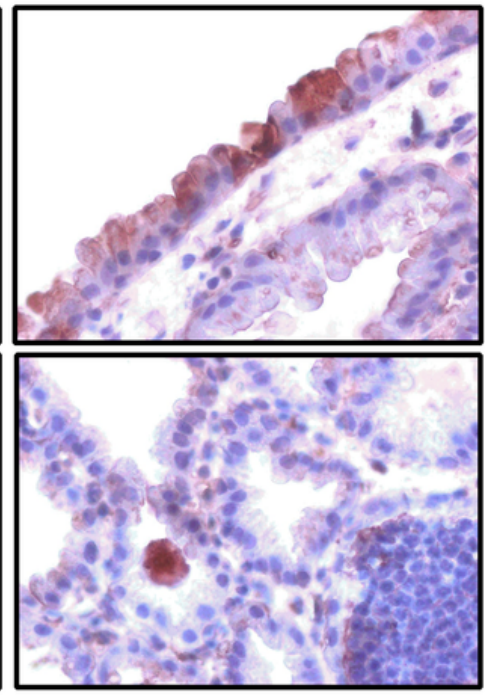

C

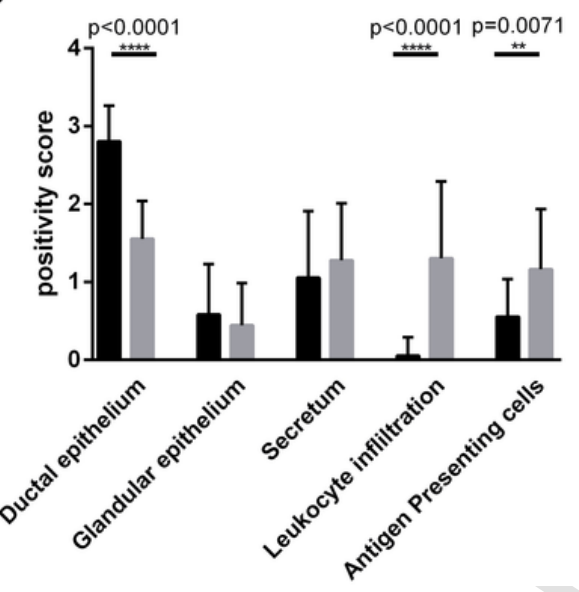

D

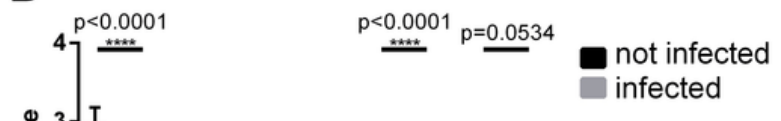

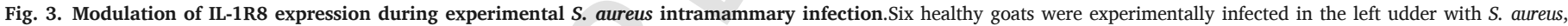

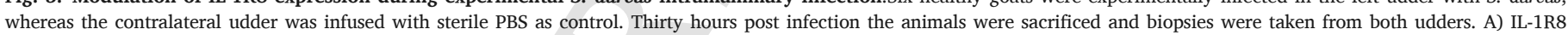

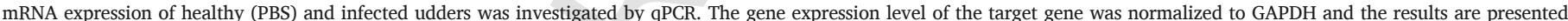

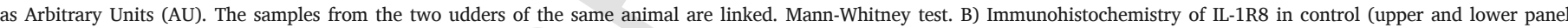

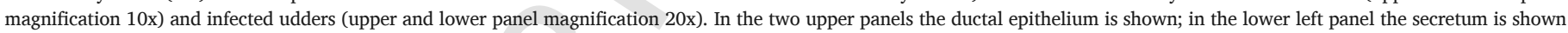

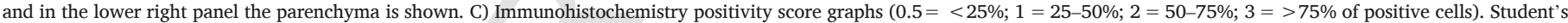
T or Mann-Whitney test. D) Immunohistochemistry intensity score graphs ( $0.5=$ weak; $1=$ slight; $2=$ moderate; $3=$ intense). Student's T or Mann-Whitney test.
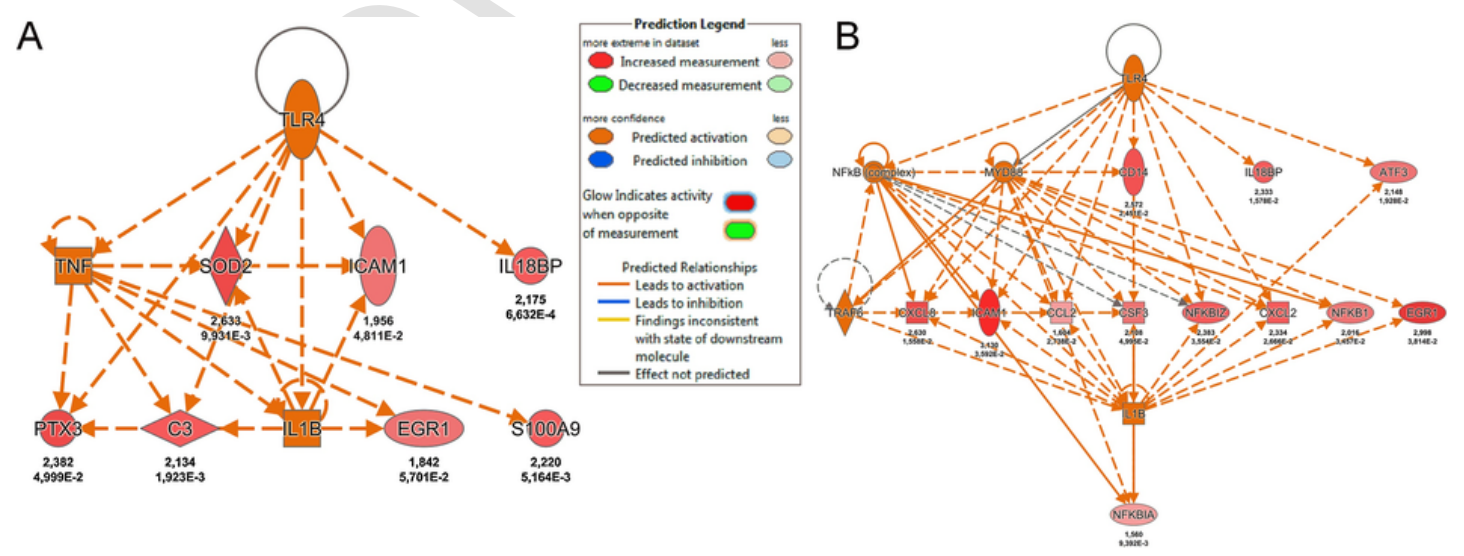

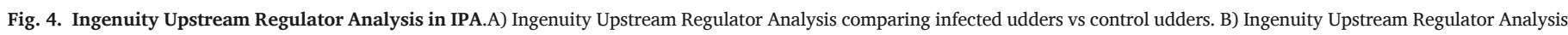
comparing infected udders vs control udders only of the 3 goats with the highest SCC count in milk. 
A

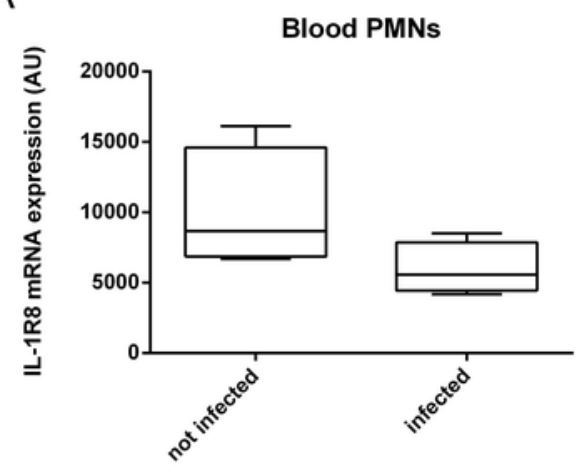

C

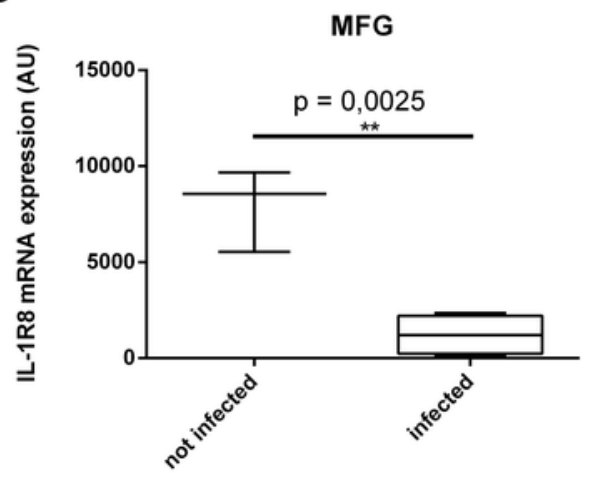

B

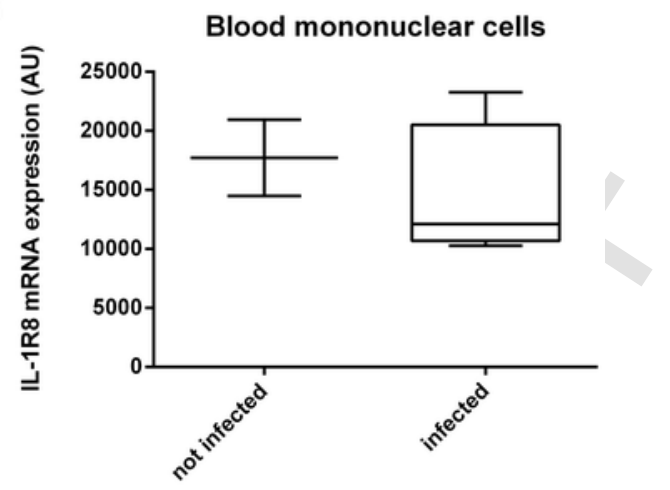

D

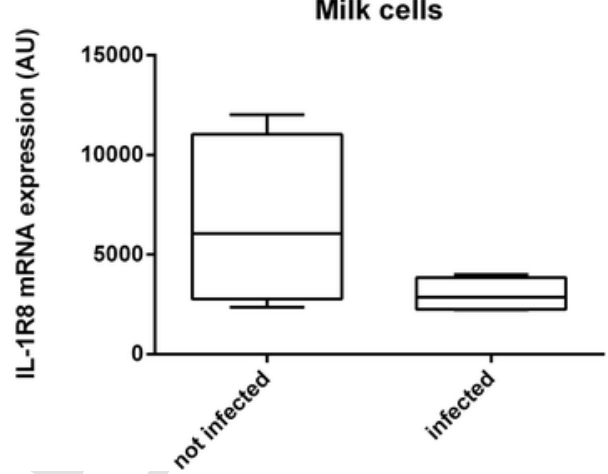

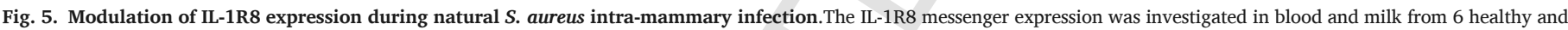

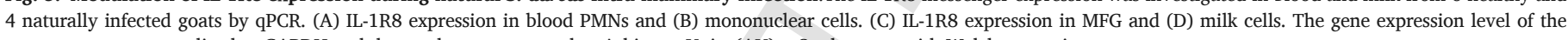
target gene was normalized to GAPDH and the results are presented as Arbitrary Units (AU). $t$ Student test with Welch correction.

IL-1R8 transcripts in different species, from human to cattle and chicken, with different patterns of expression, cellular localization and function $[35,37,45,46]$.

The sequence of the variant 1was highly homologous to the IL-1R8 messenger of other species, confirming that this molecule is highly conserved among animals. In particular, it showed high levels of homology with other ruminants, pig, and cetaceans ( $\geq 90 \%)$ compared to other mammals, chicken, zebrafish and xenopus (from 65\% to 88\%). Sheep proved the most homologous species with a 99\% of IL-1R8 homology, followed by cattle, buffalo and deer. The multiple alignment of IL-1R8 sequence of different species confirmed, again, the conservation of the molecule, suggesting that an evolutionary pressure aims to preserve its structure and function among the animals [12]. We also observed a clear sequence homology between ruminants (cow, goat, sheep, deer, and buffalo) and cetaceans (killer whale and sperm whale) based on the phylogenetic analysis of IL-1R8 sequence. The close relation between the order Cetacea (whales, dolphins) and the order Artiodactyla (cows, camels, and pigs) was previously demonstrated by paleontological [47], morphological [48] and molecular [49] studies, so that Graur et al. [49] proposed a Ruminantia/Cetacea clade.

The goat IL-1R8 gene is contained in chromosome 29 and encompasses 10 exons, similarly to humans, whereas mouse gene includes 9 exons [41].

We also investigated the pattern of expression of the molecule in a large panel of healthy goat tissues and organs. The expression of IL-1R8 in goat tissues is ubiquitous with very high levels in pancreas, followed by mammary gland and lymphoid organs (spleen and lymph nodes). The ubiquitous pattern (organs with an important epithelial component and lymphoid organs) of expression was already described in other species, but each species seems to have a peculiar pattern that maybe due to the different pathogen environment pressures [12]. The high expression of IL-1R8 in goat pancreas was already described in cattle and chicken [35,37]. Differently, other animals show the highest expression level in kidney (mouse, pig, dog) and liver (human) [12,14]. The high expression of IL-1R8 in caprine mammary gland suggests its protective role aimed to control the inflammation in an organ continuously exposed to microorganisms. The low expression of IL-1R8 in MFG confirms the immunohistological results, where the most intense and abundant positivity was observed in ductal epithelial cells compared to glandular epithelial cells. This is the region in the udder where ductal cells are constantly challenged by environmental and commensal microorganisms and do not have to trigger an inflammatory response.

The expression of IL-1R8 was locally downregulated both after experimental and natural infection respectively in ductal epithelial cells and MFG. The down-modulation of IL-1R8 is in agreement with previous data $[14,17,27,50]$. Interestingly, we observed an up-regulation in the expression of the receptor in leukocytes infiltrating the infected tissues (mainly macrophages or DCs). The up-regulation of IL-1R8 in macrophages and DCs could impair the innate immune response in the udder against $S$. aureus, leading to a failed clearance of the pathogen, the establishment of a chronic infection and severe tissue damage. The upregulation of IL-1R8 by microorganisms has been already demonstrated previously [51]. The up-regulation of IL-1R8 could be directly caused by the pathogen as a strategy to escape the immune system or indirectly by the effect of inflammatory mediators induced by the $S$. aureus (IL-10, prostaglandins).

The IPA analysis predicted the activation of TLR4 pathway in the infected tissues compared to the controls. The activation of TLR4 pathway involves the activation of signaling molecules (MyD88 and TRAF6), transcription factors such as NFkB and inflammatory related molecules such as cytokines (IL-1 $\beta$ and TNF $\alpha$ ), adhesion molecules (ICAM), chemokines (CXCL8, CCL2, CXCL2), pentraxins (PTX3) and 
complement family members (C3). This activation could be partly due to the downregulation of IL-1R8 in epithelial cells [37].

\section{Conclusions}

Our results suggest an important role of IL-1R8 in the response of mammary gland to $S$. aureus infection. Indeed, it could represent a target for an immunotherapy in the fight against mastitis allowing for the reduction of antibiotic therapy. Further studies are needed to better clarify the role of IL-1R8 in the mammary gland immune response and accurately define which are the main stimuli and mechanisms that up-regulate the orphan receptor in macrophages or DCs and downregulate it in the epithelial cells.

\section{Credit author statement}

Contribution: F.R., J.F., V.B., and P.M.. conceived the study; F.R., J.F., P.R., and B.C. designed and/or conducted experiments, performed data analysis and interpretation, and informed study direction; V.B. and G.C., helped with experimental work; F.R. and B.C. performed bioinformatics analyses; F.R., V.B., B.S., D.V. and P.M. drafted the manuscript; V.H. helped in review and editing the manuscript; and all authors discussed the results and commented on the manuscript; B.S. edited the English of the manuscript.

\section{Funding}

This work was supported by the Piano Sviluppo UNIMI 2014 Project, Linea 2 of the University of Milan (15-6-3026000-1000).

\section{Declaration of Competing Interest}

None.

\section{Acknowledgment}

The authors wish to thank Prof. Cavalchini to allow us the collection of milk and blood samples from the animals of his farm.

\section{Appendix A. Supplementary data}

Supplementary material related to this article can be found, in the online version, at doi:https://doi.org/10.1016/j.cimid.2019.101339.

\section{References}

[1] J.R. Middleton, A. Saeman, L.K. Fox, J. Lombard, J.S. Hogan, K.L. Smith, The National Mastitis Council: A Global Organization for Mastitis Control and Milk Quality, 50 Years and BeyondJ Mammary Gland Biol, J. Mammary Gland Biol. Neoplasia 19 (3-4) (2014) 241-251.

[2] D. Bergonier, R. de Cremoux, R. Rupp, G. Lagriffoul, X. Berthelot, Mastitis of dairy small ruminants, Vet. Res. 34 (5) (2003) 689-716.

[3] D.D. Bannerman, M.J. Paape, J.W. Lee, X. Zhao, J.C. Hope, P. Rainard, Escherichia coli and Staphylococcus aureus elicit differential innate immune responses following intramammary infection, Clin. Diagn. Lab. Immunol. 11 (3) (2004) 463-472.

[4] H. Lahouassa, E. Moussay, P. Rainard, C. Riollet, Differential cytokine and chemokine responses of bovine mammary epithelial cells to Staphylococcus aureus and Escherichia coli, Cytokine 38 (1) (2007) 12-21.

[5] Y. Strandberg, C. Gray, T. Vuocolo, L. Donaldson, M. Broadway, R. Tellam, Lipopolysaccharide and lipoteichoic acid induce different innate immune responses in bovine mammary epithelial cells, Cytokine 31 (1) (2005) 72-86.

[6] C.A. Dinarello, Overview of the IL-1 family in innate inflammation and acquired immunity, Immunol. Rev. 281 (1) (2018) 8-27.

[7] A. Bowie, L.A. O'Neill, The interleukin-1 receptor/Toll-like receptor superfamily: signal generators for pro-inflammatory interleukins and microbial products, J. Leukoc. Biol. 67 (4) (2000) 508-514.

[8] K. Takeda, S. Akira, TLR signaling pathways, Semin. Immunol. 16 (1) (2004) 3-9.

[9] A. Mantovani, M. Locati, A. Vecchi, S. Sozzani, P. Allavena, Decoy receptors: a strategy to regulate inflammatory cytokines and chemokines, Trends Immunol. 22 (6) (2001) 328-336.
[10] C. Garlanda, C.A. Dinarello, A. Mantovani, The interleukin-1 family: back to the future, Immunity 39 (6) (2013) 1003-1018.

[11] M. Molgora, I. Barajon, A. Mantovani, C. Garlanda, Regulatory role of IL-1R8 in immunity and disease, Front. Immunol. 7 (2016) 149.

[12] F. Riva, N. Polentarutti, G. Tribbioli, A. Mantovani, C. Garlanda, L. Turin, The expression pattern of TIR8 is conserved among vertebrates, Vet. Immunol. Immunopathol. 131 (1-2) (2009) 44-49.

[13] A. Anselmo, F. Riva, S. Gentile, C. Soldani, M. Barbagallo, C. Mazzon, F. Feruglio, N. Polentarutti, P. Somma, P. Carullo, C. Angelini, M. Bacci, G.L. Mendolicchio, A. Voza, M. Nebuloni, A. Mantovani, C. Garlanda, Expression and function of IL-1R8 (TIR8/SIGIRR): a regulatory member of the IL-1 receptor family in platelets, Cardiovasc. Res. 111 (4) (2016) 373-384.

[14] N. Polentarutti, G.P. Rol, M. Muzio, D. Bosisio, M. Camnasio, F. Riva, C. Zoja, A. Benigni, S. Tomasoni, A. Vecchi, C. Garlanda, A. Mantovani, Unique pattern of expression and inhibition of IL-1 signaling by the IL-1 receptor family member TIR8/ SIGIRR, Eur. Cytokine Netw. 14 (4) (2003) 211-218.

[15] F.M. Batliwalla, W. Li, C.T. Ritchlin, X. Xiao, M. Brenner, T. Laragione, T. Shao, R. Durham, S. Kemshetti, E. Schwarz, R. Coe, M. Kern, E.C. Baechler, T.W. Behrens, P.K. Gregersen, P.S. Gulko, Microarray analyses of peripheral blood cells identifies unique gene expression signature in psoriatic arthritis, Mol Med 11 (1-12) (2005) 21-29.

[16] R. Gopal, D. Birdsell, F.P. Monroy, Regulation of toll-like receptors in intestinal epithelial cells by stress and Toxoplasma gondii infection, Parasite Immunol. 30 (11-12) (2008) 563-576.

[17] C. Kadota, S. Ishihara, M.M. Aziz, M.A. Rumi, N. Oshima, Y. Mishima, I. Moriyama, T. Yuki, Y. Amano, Y. Kinoshita, Down-regulation of single immunoglobulin interleukin-1R-related molecule (SIGIRR)/TIR8 expression in intestinal epithelial cells during inflammation, Clin. Exp. Immunol. 162 (2) (2010) 348-361.

[18] J.C. Leemans, L.M. Butter, G.J. Teske, I. Stroo, W.P. Pulskens, S. Florquin, The toll interleukin-1 receptor (IL-1R) 8/single Ig domain IL-1R-related molecule modulates the renal response to bacterial infection, Infect. Immun. 80 (11) (2012) 3812-3820.

[19] D. Li, X. Zhang, B. Chen, SIGIRR participates in negative regulation of LPS response and tolerance in human bladder epithelial cells, BMC Immunol. 16 (2015) 73.

[20] N. Nanthakumar, D. Meng, A.M. Goldstein, W. Zhu, L. Lu, R. Uauy, A. Llanos, E.C. Claud, W.A. Walker, The mechanism of excessive intestinal inflammation in necrotizing enterocolitis: an immature innate immune response, PLoS One 6 (3) (2011), e17776.

[21] B. Ragnarsdottir, M. Samuelsson, M.C. Gustafsson, I. Leijonhufvud, D. Karpman, C Svanborg, Reduced toll-like receptor 4 expression in children with asymptomatic bacteriuria, J. Infect. Dis. 196 (3) (2007) 475-484.

[22] T. Véliz Rodriguez, F. Moalli, N. Polentarutti, M. Paroni, E. Bonavita, A. Anselmo, M. Nebuloni, S. Mantero, S. Jaillon, A. Bragonzi, A. Mantovani, F. Riva, C. Garlanda, Role of Toll interleukin-1 receptor (IL-1R) 8, a negative regulator of IL-1R/ Toll-like receptor signaling, in resistance to acute Pseudomonas aeruginosa lung infection, Infect. Immun. 80 (1) (2012) 100-109.

[23] K. Bulek, S. Swaidani, J. Qin, Y. Lu, M.F. Gulen, T. Herjan, B. Min, R.A. Kastelein M. Aronica, M. Kosz-Vnenchak, X. Li, The essential role of single Ig IL-1 receptor-related molecule/Toll IL-1R8 in regulation of Th2 immune response, J. Immunol. 182 (5) (2009) 2601-2609.

[24] J. Qin, Y. Qian, J. Yao, C. Grace, X. Li, SIGIRR inhibits interleukin-1 receptor- and toll-like receptor 4-mediated signaling through different mechanisms, J. Biol. Chem. 280 (26) (2005) 25233-25241.

[25] M. Lech, C. Garlanda, A. Mantovani, C.J. Kirschning, D. Schlondorff, H.J. Anders, Different roles of TiR8/Sigirr on toll-like receptor signaling in intrarenal antigen-presenting cells and tubular epithelial cells, Kidney Int. 72 (2) (2007) 182-192.

[26] M. Lech, O.P. Kulkarni, S. Pfeiffer, E. Savarese, A. Krug, C. Garlanda, A. Mantovani, H.J. Anders, Tir8/Sigirr prevents murine lupus by suppressing the immunostimulatory effects of lupus autoantigens, J. Exp. Med. 205 (8) (2008) 1879-1888.

[27] D. Wald, J. Qin, Z. Zhao, Y. Qian, M. Naramura, L. Tian, J. Towne, J.E. Sims, G.R. Stark, X. Li, SIGIRR, a negative regulator of Toll-like receptor-interleukin 1 receptor signaling, Nat. Immunol. 4 (9) (2003) 920-927.

[28] C. Garlanda, F. Riva, N. Polentarutti, C. Buracchi, M. Sironi, M. De Bortoli, M. Muzio, R. Bergottini, E. Scanziani, A. Vecchi, E. Hirsch, A. Mantovani, Intestinal inflammation in mice deficient in Tir8, an inhibitory member of the IL-1 receptor family, Proc. Natl. Acad. Sci. U. S. A. 101 (10) (2004) 3522-3526.

[29] J. Gong, T. Wei, R.W. Stark, F. Jamitzky, W.M. Heckl, H.J. Anders, M. Lech, S.C. Rossle, Inhibition of Toll-like receptors TLR4 and 7 signaling pathways by SIGIRR: a computational approach, J. Struct. Biol. 169 (3) (2010) 323-330.

[30] M.F. Gulen, Z. Kang, K. Bulek, W. Youzhong, T.W. Kim, Y. Chen, C.Z. Altuntas, K. Sass Bak-Jensen, M.J. McGeachy, J.S. Do, H. Xiao, G.M. Delgoffe, B. Min, J.D. Powell, V.K. Tuohy, D.J. Cua, X. Li, The receptor SIGIRR suppresses Th17 cell proliferation via inhibition of the interleukin-1 receptor pathway and mTOR kinase activation, Immunity 32 (1) (2010) 54-66.

[31] H. Xiao, M.F. Gulen, J. Qin, J. Yao, K. Bulek, D. Kish, C.Z. Altuntas, D. Wald, C Ma, H. Zhou, V.K. Tuohy, R.L. Fairchild, C. de la Motte, D. Cua, B.A. Vallance, X. $\mathrm{Li}$, The Toll-interleukin-1 receptor member SIGIRR regulates colonic epithelial homeostasis, inflammation, and tumorigenesis, Immunity 26 (4) (2007) 461-475.

[32] P. Cremonesi, R. Capoferri, G. Pisoni, M. Del Corvo, F. Strozzi, R. Rupp, H. Caillat, P. Modesto, P. Moroni, J.L. Williams, B. Castiglioni, A. Stella, Response of the goat mammary gland to infection with Staphylococcus aureus revealed by gene expres- 
sion profiling in milk somatic and white blood cells, BMC Genomics 13 (2012) 540.

[33] A.F. Hope, Laboratory Handbook on Bovine Mastitis, 3rd ed., 2017.

[34] J. Filipe, G. Curone, V. Bronzo, G. Pisoni, P. Cremonesi, C. Pollera, L. Turin, D. Vigo, P. Roccabianca, M. Caniatti, P. Moroni, F. Riva, Pentraxin 3 is up-regulated in epithelial mammary cells during Staphylococcus aureus intra-mammary infection in goat, Comp. Immunol. Microbiol. Infect. Dis. 59 (2018) 8-16.

[35] L. Turin, G. Manarolla, F. Riva, Expression of TIR8 receptor in chicken tissues, J. Biol. Regul. Homeost. Agents 28 (3) (2014) 515-522.

[36] E. Trevisi, M. Amadori, F. Riva, G. Bertoni, P. Bani, Evaluation of innate immune responses in bovine forestomachs, Res. Vet. Sci. 96 (1) (2014) 69-78.

[37] F. Riva, M.M. Rahman, L. Turin, F. Ceciliani, S. Russo, G. Tribbioli, C. Lecchi, TIR8 receptor expression in bovine tissues, Vet. Immunol. Immunopathol. 136 (1-2) (2010) 65-70.

[38] M. Bonnet, L. Bernard, S. Bes, C. Leroux, Selection of reference genes for quantitative real-time PCR normalisation in adipose tissue, muscle, liver and mammary gland from ruminants, Animal 7 (8) (2013) 1344-1353.

[39] P. Modesto, S. Peletto, G. Pisoni, P. Cremonesi, B. Castiglioni, S. Colussi, M. Caramelli, V. Bronzo, P. Moroni, P.L. Acutis, Evaluation of internal reference genes for quantitative expression analysis by real-time reverse transcription-PCR in somatic cells from goat milk, J. Dairy Sci. 96 (12) (2013) 7932-7944.

[40] A. Dereeper, V. Guignon, G. Blanc, S. Audic, S. Buffet, F. Chevenet, J.F. Dufayard, S. Guindon, V. Lefort, M. Lescot, J.M. Claverie, O. Gascuel, Phylogeny.fr: robust phylogenetic analysis for the non-specialist, Nucleic Acids Res. 36 (2008) W465-469, (Web Server issue).

[41] F. Riva, E. Bonavita, E. Barbati, M. Muzio, A. Mantovani, C. Garlanda, TIR8/SIGIRR is an Interleukin-1 Receptor/Toll like receptor family member with regulatory functions in inflammation and immunity, Front. Immunol. 3 (2012) 322.
[42] M.A. Anwar, S. Basith, S. Choi, Negative regulatory approaches to the attenuation of Toll-like receptor signaling, Exp. Mol. Med. 45 (2013) e11.

[43] R. Bonecchi, C. Garlanda, A. Mantovani, F. Riva, Cytokine decoy and scavenger receptors as key regulators of immunity and inflammation, Cytokine 87 (2016) 37-45.

[44] O. Shibolet, D.K. Podolsky, TLRs in the Gut. IV. Negative regulation of Toll-like receptors and intestinal homeostasis: addition by subtraction, Am. J. Physiol. Gastrointest. Liver Physiol. 292 (6) (2007) G1469-73.

[45] M.G. Vilia, M. Tocchetti, E. Fonte, I. Sana, M. Muzio, Characterization of a long isoform of IL-1R8 (TIR8/SIGIRR), Eur. Cytokine Netw. 28 (2) (2017) 63-69.

[46] J. Zhao, K. Bulek, M.F. Gulen, J.A. Zepp, G. Karagkounis, B.N. Martin, H. Zhou, M. Yu, X. Liu, E. Huang, P.L. Fox, M.F. Kalady, S.D. Markowitz, X. Li, Human Colon tumors express a dominant-negative form of SIGIRR that promotes inflammation and colitis-associated Colon Cancer in mice, Gastroenterology 149 (7) (2015) 1860-1871, e8.

[47] P.D. Gingerich, B.H. Smith, E.L. Simons, Hind limbs of eocene basilosaurus: evidence of feet in whales, Science 249 (4965) (1990) 154-157.

[48] M.J. Novacek, Mammalian phylogeny: shaking the tree, Nature 6 (6365) (1992) $121-125$.

[49] D. Graur, D.G. Higgins, Molecular evidence for the inclusion of cetaceans within the order Artiodactyla, Mol. Biol. Evol. 11 (3) (1994) 357-364.

[50] X. Huang, L.D. Hazlett, W. Du, R.P. Barrett, SIGIRR promotes resistance against Pseudomonas aeruginosa keratitis by down-regulating type-1 immunity and IL-1R1 and TLR4 signaling, J. Immunol. 177 (1) (2006) 548-556.

[51] W. Wu, Y. Wang, J. Zou, F. Long, H. Yan, L. Zeng, Y. Chen, Bifidobacterium adolescentis protects against necrotizing enterocolitis and upregulates TOLLIP and SIGIRR in premature neonatal rats, BMC Pediatr. 17 (1) (2017) 1. 